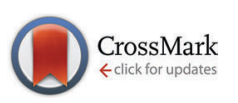

Cite this: Phys. Chem. Chem. Phys., 2016, 18, 10647

DOI: $10.1039 / c 6 c p 90084 d$

www.rsc.org/pccp

\section{Correction: Reversibility and two state behaviour in the thermal unfolding of oligomeric TIM barrel proteins}

\author{
Sergio Romero-Romero, ${ }^{a}$ Miguel Costas, ${ }^{b}$ Adela Rodríguez-Romeroc and \\ D. Alejandro Fernández-Velasco*a
}

Correction for 'Reversibility and two state behaviour in the thermal unfolding of oligomeric TIM barrel proteins' by Sergio Romero-Romero et al., Phys. Chem. Chem. Phys., 2015, 17, 20699-20714.

The authors would like to correct an error on page 20706 of the article, left column, fourth line from bottom. The correct sentence should read as follows:

The size of the biggest cavity, located at the interface between $\beta$-strands $4-6$ and $\alpha$-helices 4-6 (comprising residues 90-120, 145-160 and 195-200; Fig. S6, ESI ), is the main responsible of the cavity volume difference between RevTIMs and IrrevTIMs (Table 4 and Fig. 7).

The Royal Society of Chemistry apologises for these errors and any consequent inconvenience to authors and readers.

\footnotetext{
${ }^{a}$ Laboratorio de Fisicoquímica e Ingeniería de Proteínas, Departamento de Bioquímica, Facultad de Medicina, Universidad Nacional Autónoma de México, 04510 Ciudad de México, Distrito Federal, Mexico.E-mail: fdaniel@unam.mx

${ }^{b}$ Laboratorio de Biofisicoquímica, Departamento de Fisicoquímica, Facultad de Química, Universidad Nacional Autónoma de México, 04510 Ciudad de México, Distrito Federal, Mexico

${ }^{c}$ Laboratorio de Química de Biomacromoléculas 3, Departamento de Química de Biomacromoléculas, Instituto de Química, Universidad Nacional Autónoma de México, 04510 Ciudad de México, Distrito Federal, Mexico
} 